\title{
The effect of 3-D orientation and stretches on the detection of dotted planes
}

\author{
WILFRED M. KINCAID \\ University of Michigan, Ann Arbor, Michigan \\ and \\ WILLIAM R. UTTAL \\ Naval Ocean Systems Center, Kailua, Hawaii
}

\begin{abstract}
The present experiment used a masking procedure in which a dotted stimulus form was masked by random dots to explore human form-detection capabilities. The dotted stimulus forms were located in a stereoscopically generated three-dimensional rectangular volume. The form was defined by a set of randomly positioned dots always restricted to a plane. The orientation of the dotted plane varied from frontoparallel to diagonal around a vertical axis of rotation passing through the center of the rectangular volume. The plane always stretched across the region from sidewall to sidewall. Since the volume was deeper than it was wide, both the apparent area and the apparent dot density of the plane varied with orientation. The masking dots were distributed at random throughout the volume. For a constant number of stimulus-form and masking dots, however, the detectability of the plane was shown not to depend upon orientation. This counterintuitive result in conjunction with earlier findings suggests that there is a range of conditions over which detectability depends only on the total numbers of stimulus and masking dots and not upon subjective orientation, density, or area of the plane. Therefore, observers respond as if they were sensitive to the retinal two-dimensional density rather than the apparent threedimensional density.
\end{abstract}

This report is concerned with dotted stimulus-form detection when that form is masked or hidden by random "visual noise" dots. The question asked is: Does the detectability of the stimulus form, which is known to be strongly affected by the actual physical density of dots, vary as a function of the apparent density? Apparent density in this case is manipulated by stereoscopically "stretching" a planar stimulus form into a threedimensional shape of greater apparent area than that possessed by a prototype frontoparallel plane. This is accomplished by rotating the plane to different angles inside a rectangular rather than a cubical volume, as shown in Figure 1. As the plane is rotated, it is kept anchored to the walls of the rectangular space, thus increasing its apparent surface area. The two-dimensional projection of this increased three-dimensional area remains constant, however. In other words, we ask whether psychophysical detectability will follow the retinal, two-dimensional projection, which remains relatively nearly constant, or the apparent surface area in three-dimensional space, which varies significantly as the stimulus form is rotated.

This research was supported by the Office of Naval Research under Contract N00014-81-C-0366 to the University of Michigan. John J. O'Hare was the Scientific Officer. W. M. Kincaid's mailing address is: Department of Mathematics, 3220 Angell Hall, The University of Michigan, Ann Arbor, MI 48109. Reprint requests should be addressed to W. R. Uttal, at Naval Ocean Systems Center, Hawaii Laboratory, Box 997, Kailua, HI 96734.
In a previous study (Uttal, Fitzgerald, \& Eskin, 1975), square planes of $4.4^{\circ} \times 4.4^{\circ}$ in subtense were placed centrally in a cubical, rather than a rectangular, volume. The planes were either placed in a frontoparallel position or rotated from that position about the $y$ (vertical) axis through the center of the cube. Surprisingly, no effect of rotation on detection was observed except for planes rotated to within a degree or two of the edge-on $\left(90^{\circ}\right)$ position. In an extension of the experiment (Uttal, 1983), rotations about a variety of other axes and combinations of other axes yielded similar negative results.

More recently, Uttal (1984) compared planar dotted surfaces with nonplanar curved ones. The planes were frontoparallel, centrally placed, and of the same $5.4^{\circ}$ height and width as the cube. The curved surfaces were portions of cylinders, hemispheres, parabolic arches, and other forms generated from a standard polynomial generating rule. Quite unexpectedly, the probability of correct responses (for fixed numbers of stimulus and noise points) decreased only slightly with increasing departure from the plane for each class of surface. In fact, many of the most extremely distorted nonplanar surfaces showed no significant differences in detection scores from the planar stimulus. It was only when the surface had more than one maximum or minimum (in depth) that barely significant effects were obtained.

One a priori reason for having expected effects of form is that the perceived surface areas of the apparently three- 


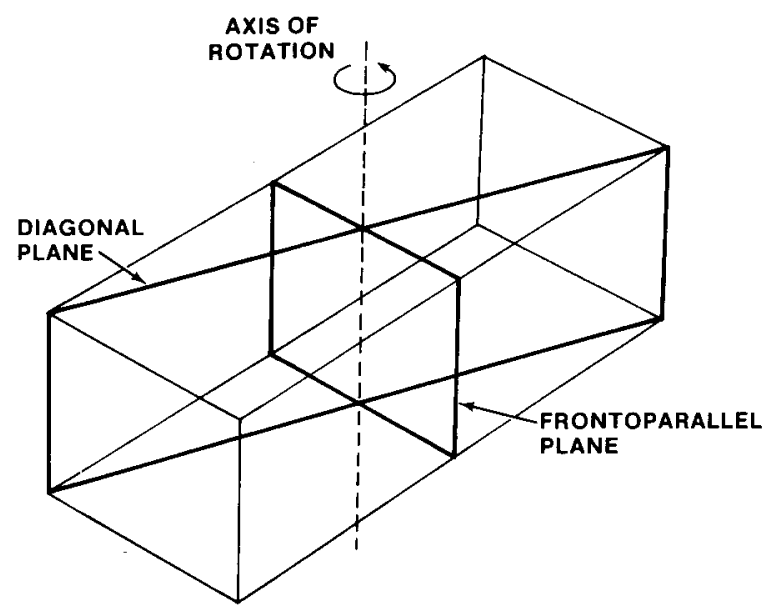

Figure 1. Diagrammatic sketch showing the manner in which the apparent surface area of a stereoscopically generated plane varies as it is rotated to different orientations in the rectangular viewing space.

dimensional curved surfaces are greater than the apparent area of the plane. Given that the same number of dots was used in each case, the apparent density should be effectively less for the curved surfaces than for the plane. Thus, detectability should have decreased even if the density of the masking dots remained constant. The experimental results thus suggest that the stereoscopically constructed third (depth) dimension, which has to be indirectly calculated from invariances in the disparity information, is not processed the same way in this context as are the other two dimensions, which are physically projected onto the retina. A suggested counterargument to this line of reasoning is that the observer might be selectively directing most of his attention to restricted subportions of the curved surface that are nearly frontoparallel, where the densities remain locally constant.

The issue is important enough for us to have judged it desirable to seek further experimental evidence concerning the effect of apparent, as opposed to real, density on detectability. To this end, we noted that comparing a frontoparallel plane with a tilted plane (instead of with a curved surface) would avoid the local region complication as well as other potential artifacts that might be associated with curvature as such.

Uttal et al.'s (1975) experiment involved such a comparison. However, in that experiment the frontal projection area of the plane was controlled so that it varied with the angle of rotation; thus, the density of the dots of the stimulus form was constant on the apparent plane regardless of its orientation but varied in the frontal projection. This difference makes it impossible to settle the issue raised here with these data. Accordingly, we carried out the experiment described below. The main differences were that a regular parallelepiped (rather than a cubical volume) was used as the viewing space and the sides of the rectangular plane were anchored to the sides of the space rather than restricted to original dimensions of the frontoparallel plane.

\section{METHOD}

Four observers, undergraduate students, were used in this experiment. Although there were some differences among the individuals, all displayed the same general pattern of results as a function of the variables of the experiment.

The equipment used for generating stimuli has been described elsewhere (Uttal, 1983, 1984). To recapitulate briefly, the images for the right and left eyes were presented on the right and left halves, respectively, of a single split-screen oscilloscope (Hewlett-Packard 1311-B) coated with a high-speed P-24 phosphor. The observer viewed the two images through binocular rotary prisms that were individually adjusted at the beginning of each session for comfortable convergence. An opaque septum extended from the binocular prisms to the screen of the oscilloscope (a distance of $71 \mathrm{~cm}$ ) and thus divided the two halves of the screen so that neither eye saw the field of view of the other. For the observer, the stimulus took on the highly realistic appearance of a three-dimensional configuration of points of light. (The visual angle subtended by each dot was below the normal point spread function of the eye.) All the stimuli were confined to a rectangular region subtending $3^{\circ}$ horizontally and vertically and having an apparent depth of about $5.4^{\circ}$ (corresponding to a range of $28^{\prime}$ of visual angle disparities). These dimensions define a rectangular parallelepiped (with a square frontoparallel cross-section) rather than the $5.4^{\circ} \times 5.4^{\circ} \times 5.4^{\circ}$ cube used previously.

The sequence of visible events in each trial was as follows. First, a pair of dots (one for each eye), so placed as to provide a stereoscopically fused single fixation-convergence dot at the center of the rectangular region described above, was illuminated for $1 \mathrm{sec}$. Immediately following the fixation-convergence dot, the first of the two alternative presentations was displayed for $1 \mathrm{sec}$. This was followed by another 1-sec display of the fixation-convergence dot, and then by the display of the other alternative stimulus presentation. One of the stimulus presentations consisted of a set of stimulus dots arranged in a plane and a variable number of masking dots. The other consisted of the same number and arrangement of masking dots plus a set of "dummy" dots. Because the plane was originally defined by a set of random dots, this procedure guarantees that there is no way that the dummy dots could be phenomenally distinguished from the stimulus plane except by virtue of the three-dimensional organization. The dummy dots had the same $x$ and $y$ coordinates as those in the stimulus plane, but differed only in that they had randomly chosen $z$ (depth) coordinates rather than a set limited to the planar surface.

Following the second presentation, the screen remained dark until the observer depressed one of two hand-held pushbuttons to indicate which of the two presentations he thought most likely to have included the planar stimulus form. At that point, a "plus" or a "minus," indicating that either a correct or an incorrect choice had been made, appeared on the oscilloscope. The cycle was then repeated. Thus, this experiment used a two-alternative, forced-choice procedure, and the probability of a correct response ranged from a minimum of $50 \%$ to a maximum of $100 \%$.

In this experiment, the dots of the stimulus form in each presentation lay in a single vertical plane passing through the center of the rectangular stimulus region. In any trial, the plane could be oriented to any one of 10 possible positions, ranging from frontoparallel to diagonal. In the frontoparallel version, the plane extended across the width of the rectangular viewing space. In the diagonal position, the plane extended from the left front edge to the right rear edge of the stimulus region. In all cases, the plane extended completely across the rectangular viewing space; that is, its top and bottom edges lay in the top and bottom surfaces of the 
stimulus region and its left and right edges were anchored to the left and right sides of the stimulus region. Thus, the dots lay in a rectangle whose height was $3^{\circ}$ and whose width varied from $3^{\circ}$ to $\left(3^{2}+5.4^{2}\right)^{1 / 2}$ degrees, or $6.18^{\circ}$. Hence, the diagonal plane had 2.06 times the apparent area of the frontoparallel one. Of course, the retinal projections of the left- and right-eye images remained nearly constant.

The experiment was carried out in 14 successive sessions. During the first 7 days, the masking-dot density was progressively increased from 25 to 200 dots in the following order: $25,50,100$, $125,150,175$, and 200 . During the second 7 days, the maskingdot density was decreased in reverse order.

Thirty distinctly different planar stimuli were used in this study: 10 with 25,10 with 36 , and 10 with 49 randomly positioned dots. Ten different orientations were used, varying from a frontoparallel plane to an orientation of $64.1^{\circ}$ (a diagonal from the front corner of the rectangular space to the rear corner) in equal angular steps of $7.12^{\circ}$.

\section{RESULTS}

As in previous experiments, and as shown in Figure 2, detectability improved with increased numbers of dots in the planar stimulus form and declined with increased numbers of masking dots. This is the effect of the signal-tonoise ratio of the real dot densities. Of primary interest in this study, however, is the relation between detectability and the apparent orientation and density of the plane. This outcome is plotted in Figure 3 for the seven different masking-dot densities. In this figure, the results for 25,36 , and 49 stimulus-form dot densities are pooled. It is immediately apparent that any effect of orientation upon detectability must be extremely small. (No statistical analysis is necessary here. The absence of a significant effect would not lead one to believe that the effect was literally zero, while some low level of significance, if present, would not alter the conclusion that the effect was very small.)

\section{DISCUSSION}

These new results, obtained with planes of various apparent areas, resemble those obtained for curved stimulus surfaces in showing insensitivity to departures of the surface from a frontoparallel plane. As remarked earlier, questions that can be raised in connection with the curved surfaces do not apply to the present case. These new results are thus more clear-cut in their implications. Since the apparent density of the dots in the stimulus plane varied by more than 2 to 1 (for a given number of dots) as the plane shifted from the frontoparallel to the diagonal position, one would have expected to observe reduced detectability as the plane tilted away from the frontoparallel position. Furthermore, in the diagonal position, the masking dots are, on the average, closer to the plane than when the plane is in the frontoparallel position. This also suggests, according to some points of view, that the masking should be greater in the former than in the latter positions. The lack of such a trend in either case suggests that the apparent third dimension does not combine with the other two in Euclidean fashion in this context.

The current results are similar, at first examination, to those obtained in the experiment with rotated planes cited earlier (Uttal et al., 1975); in both cases, the effect of changing orientation was conspicuous by its absence. When the experimental conditions are compared, however, this apparent agreement is seen to require further examination. In the earlier experiment, where there was an apparent rigid rotation of the stimulus plane, the observed constancy was in accord with predictions based

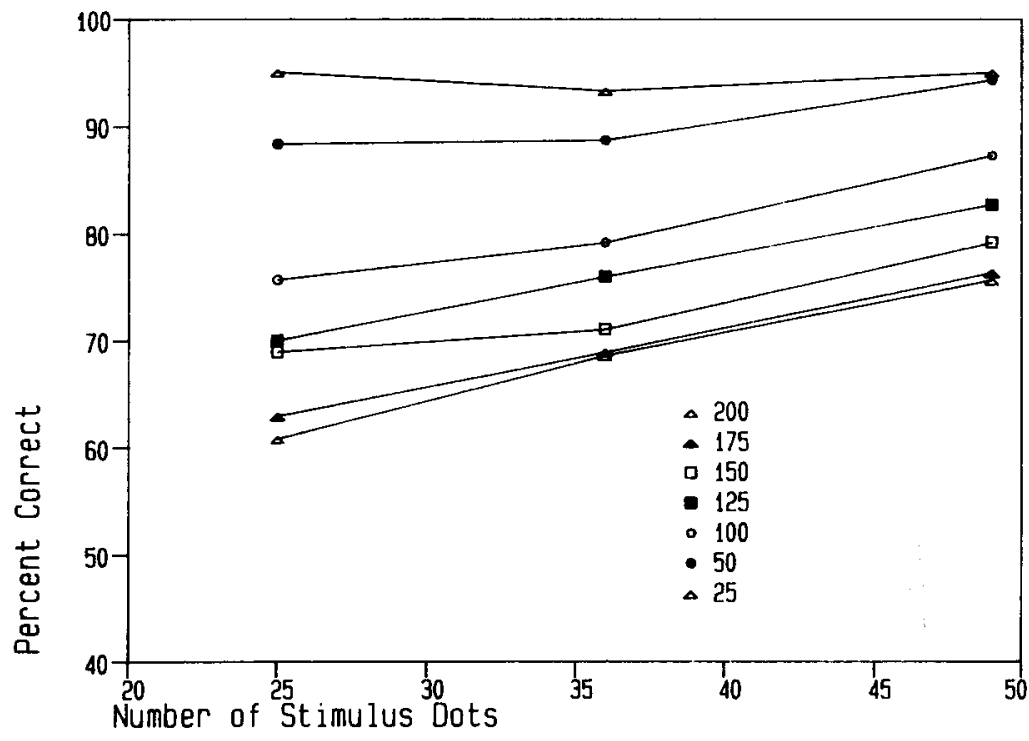

Figure 2. The effect of the number of dots in the stimulus plane on its detectability. The different curves are parametric in terms of the number of masking dots. Either increasing the number of masking dots or decreasing the number of stimulus plane dots decreases detectability. 
on three-dimensional Euclidean geometry, unlike the present case. Another way of expressing the difference is to say that in the earlier experiment the projected twodimensional dot density was varied while the apparent dot density in the stimulus plane was fixed, whereas in the current experiment the reverse was the case. From this perspective, the former and current results can be reconciled most simply by assuming that apparent dot density in the stimulus plane does not affect detection (over the general range of conditions represented by these experiments) as long as the total numbers of stimulus dots and masking dots remain fixed.

This assumption is inconsistent with the general run of past results, however, and cannot be accepted without considering other differences between the experimental conditions. The most conspicuous of these differences lay in the distribution of dummy dots. This was uniform throughout the viewing space in the current experiment. In the earlier experiment (in which planes were rotated), however, the dummy dots were confined to a slab within the cubical stimulus region corresponding to the projection of the stimulus plane. (Recall that the dummy dots had the same $x$ and $y$ coordinates as the stimulus dots.) One suggestion is that the result of this distribution of dummy dots could have been to focus the observers' attention on the restricted central portion of the cube, possibly reducing the influence of masking dots outside of the slab.

This complication cannot be ignored; indeed, the desire to avoid it influenced the design of the current experi- ment. However, it seems quite unlikely that its effect depended on the angle of rotation in such a way as to just counterbalance a trend that would otherwise have been present to produce the negative result obtained here. (Recall that insensitivity to apparent angle of rotation was maintained to within a degree or two of $90^{\circ}$, that is, for a range of projected densities of 20 or more to 1.) In short, this digression points up the need for caution but furnishes no real reason for rejecting the assumption put forth above.

We are thus left with two conclusions: (1) The detectability of a surface generated by dots is insensitive to changes in the orientation or curvature of the surface as such. (2) Within a substantial, but undetermined, range of conditions, the detectability of a tilted plane depends on the total numbers of stimulus dots and masking dots, but not on either the projected or the apparent density of the stimulus dots.

This second conclusion is clearly an invitation to further research to determine its range of applicability. The most obvious approach would be along the lines of the experiment described above, using a variety of shapes and sizes of stimulus regions in place of the $3^{\circ} \times 3^{\circ} \times 5.4^{\circ}$ box employed here. By making suitable choices, one could manipulate separately the variables of projected density, apparent (three-dimensional) density in the stimulus plane, and location of masking dots relative to the plane. In this way, it could be determined to what extent the invariance observed in the experiment reported here is part of a larger picture. That it is, indeed, part of a larger picture is con-

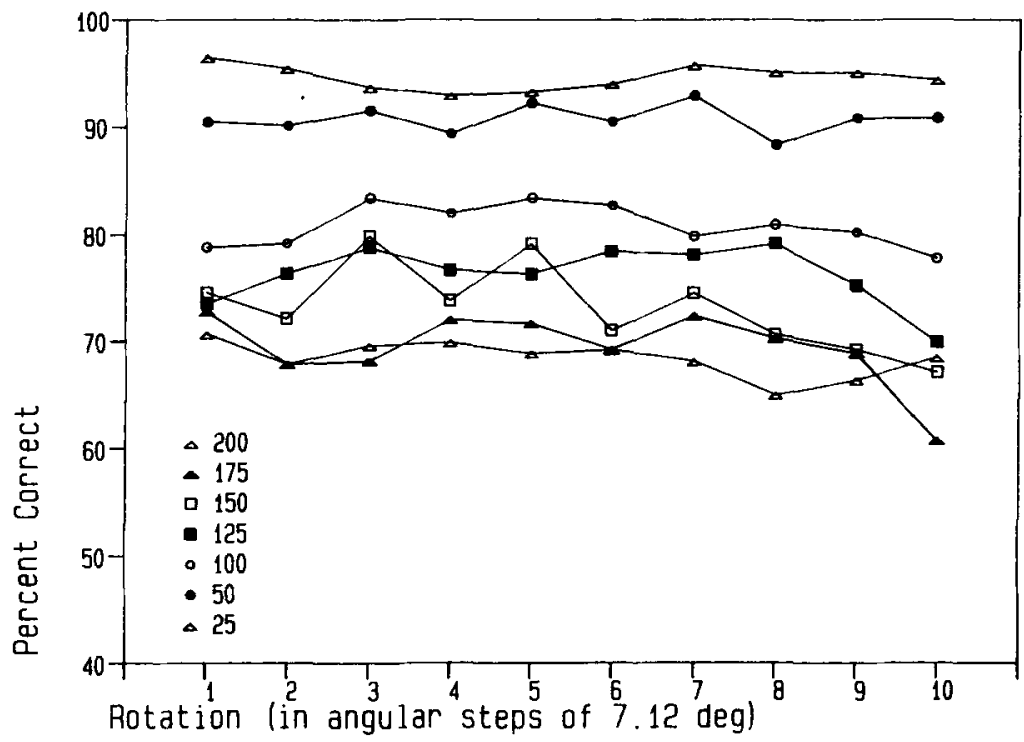

Figure 3. The negligible effect of rotating (and thus stretching the apparent surface area of the stimulus plane to various orientations in the rectangular viewing space. Despite the reduction in apparent dot density, there is little or no reduction in detectability. The numerals on the horizontal axis indicate the 10 different orientations into which the plane was rotated (1 indicates the frontoparallel orientation, and 10 indicates the diagonal orientation of maximum rotation). The different curves are parametric in terms of the maskingdot density. 
firmed by similar negative results in stereoscopic space obtained by Lappin and Langston (1984) for stereoacuity and by Julesz and Chang (1984) for their texton model.

\section{REFERENCES}

Julesz, B., \& Chang, J. J. (1984). Do hypercyclopean textons exist? Investigative Ophthalmology \& Visual Science, 25, 199.

LAPPin, J. S., LANGSTon, A., \& LiVert, D. (1984). The structure of 3-dimensional error distributions for locating points in stereoscopic space. Investigative Ophthalmology \& Visual Science, 25, 295.
UTtaL, W. R. (1983). Visual form detection in 3-dimensional space. Hillsdale, NJ: Erlbaum.

UTTAL, W. R. (1984). The detection of nonplanar surfaces in visual space. Hillsdale, NJ: Erlbaum.

UtTal, W. R., Fitzgerald, J., Eskin, T. (1975). Rotation and translation effects on stereoscopic acuity. Vision Research, 15, 939-944.

(Manuscript received November 1, 1985; revision accepted for publication April 11, 1986.) 